BMJ Open

Sport \&

Exercise

Medicine

\section{Long-standing groin pain in contact sports: a prospective case-control and MRI study}

To cite: Paajanen $\mathrm{H}$, Hermunen $\mathrm{H}$, Ristolainen $\mathrm{L}$, et al. Long-standing groin pain in contact sports: a prospective case-control and MRI study. BMJ Open Sport \& Exercise Medicine 2019;5:e000507. doi:10.1136/ bmjsem-2018-000507

Accepted 24 February 2019

Check for updates

(c) Author(s) (or their employer(s)) 2019. Re-use permitted under CC BY-NC. No commercial re-use. See rights and permissions. Published by BMJ.

${ }^{1}$ Department of Surgery, University of Eastern Finland, Kuopio, Finland

2Department of Radiology, Mikkeli Central Hospital, Mikkeli, Finland

${ }^{3}$ Department of Orthopaedics, Orton Orthopaedic Hospital, Helsinki, Finland

${ }^{4}$ Department of Radiology,

Rigshospitalet, Copenhagen, Denmark

${ }^{5}$ Arthroscopic Center Amager, Hvidovre Hospital, Copenhagen, Denmark

Correspondence to Dr Hannu Paajanen; paajanen@surffi.fi

\section{ABSTRACT}

Objectives We aimed to prospectively evaluate the prevalence of long-standing groin pain and related MRI findings in contact sports.

Methods This case-control study followed three male elite-level soccer, ice-hockey and bandy teams (102 players) for 2 years. All athletes with long-standing groin pain lasting $>30$ days and age-matched controls (1:3) from the same teams were examined clinically, using pelvic MRI and Hip and Groin Outcome Scores (HAGOS). Primary outcome measures were annual prevalence of groin pain and underlying MRI findings.

Results The annual prevalence of chronic groin pain was $7.5 \%$. Training characteristics and pain scores of athletes were similar in all teams. On MRI, there was no significant difference in the percentage of pubic bone marrow oedema ( $p=0.80$ ) between symptomatic players $(8 / 15 ; 53 \%)$ versus controls $(20 / 43 ; 47 \%)$, but adductor tendinopathy and degenerative changes at the pubic symphysis were twice more common among players with pain. Rectus muscle or iliopsoas pathology were seldom observed. Lower HAGOS subscales $(p<0.01)$ were recorded in players who experienced groin pain compared with the controls.

Conclusion Long-standing groin pain was observed annually in 1 of 14 athletes in contact sports. Abnormalities in the pubic symphysis were common MRI findings in both symptomatic and asymptomatic players.

Trial registration number NCT02560480

\section{INTRODUCTION}

In retrospective cohort studies, the prevalence of long-standing groin pain ranges between $5 \%$ and $10 \%$ in athletes and the season incidence is nearly $7 \% .^{1-3}$ Common causes include tendinopathies of adductor, iliopsoas or rectus abdominis muscles, protrusion of the posterior wall of inguinal canal ('sportsman's hernia') or impingement of the hip joint. ${ }^{1-5}$ Sometimes, a multidisciplinary investigation may reveal simultaneous multiple reasons. ${ }^{67}$ First-line management includes rest, physiotherapy, anti-inflammatory analgesics, local corticosteroid injections or seldom operative management. ${ }^{89}$

Athletes with long-standing groin pain often display characteristic MRI findings. ${ }^{310-12}$

\section{What are the findings?}

The annual prevalence of long-standing groin pain was $7.5 \%$.

- The percentage of pubic bone marrow oedema in MRI was $50 \%$ in both symptomatic and asymptomatic players, that is, it did not correlate well with groin pain.

- Other pathological MRI findings at the pubic symphysis were also common in both symptomatic and asymptomatic players.

How might it impact on clinical practice in the near future?

MRI is often used in the diagnostic workup of athletes with long-standing groin pain.

- Positive MRI findings do not necessarily correlate with long-standing groin pain.

Four main radiological abnormalities appear consistently in the symptomatic groin: degenerative changes at the pubic symphyseal joint, pathology at the adductor muscle insertion, pubic bone marrow oedema (BME) and a secondary cleft sign. ${ }^{11}$ These MRI findings may also appear in pain-free players. ${ }^{12-15}$ In some cases, only posterior inguinal wall deficiency is observed on ultrasonography. ${ }^{816} \mathrm{In}$ the present prospective case-control study, we aimed to investigate the annual prevalence of long-standing groin pain in three contact sport clubs and define the exact diagnosis behind groin symptoms using clinical investigation and MRI.

\section{METHODS}

\section{Patients}

Three elite-level male contact sport teams (soccer, ice-hockey and bandy) including 102 players were invited to join into this prospective observational case-control study. All players aged $>18$ years were followed for 2 years from August 2015 to July 2017 to find out the prevalence of long-standing groin pain (>30 days). The symptomatic athletes and their controls 


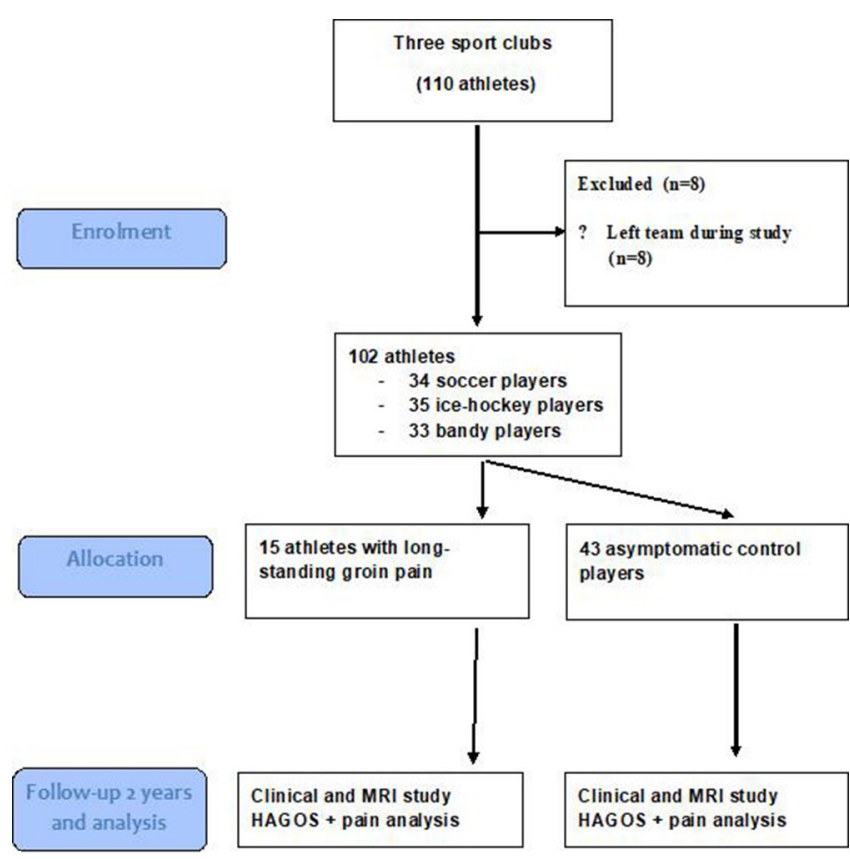

Figure 1 Flow diagram of the study. HAGOS, Hip and Groin Outcome Scores.

(ratio 1:3) matched by same sport, age and athletic exposure were included (figure 1). Control athletes were hip and groin injury free for last 6 months.

The aetiology and diagnosis of long-standing groin pain were always based on patient history, physical examination and MR imaging. The location of groin pain (visual analogue pain scores, VAS $\geq 3$; range $0-10$ ) had to be in the lower abdomen near the inguinal ligament with or without tenderness over the pubic symphysis or at the proximal insertion of adductors. Pain scores (VAS) during rest and exercise were carefully recorded at the initiation of pain and weekly after that until recovery. Clinical examination findings were divided according to Doha agreement meeting (adductor-related, inguinal-related, pubic related, iliopsoas-related, hip-related groin pain). ${ }^{16}$

The main endpoint was the annual prevalence of long-standing groin pain in each team. Other outcome measures were VAS scores and exact clinical as well as MRI findings. Hip and Groin Outcome Score (HAGOS) Questionnaire was used to determine the severity of groin injuries. ${ }^{17}$ All participants (15 symptomatic vs 43 asymptomatic control players) underwent identical standardised and reliable clinical examination, HAGOS questionnaire and MRI scans (1.5 Tesla) of the pelvis.

\section{MRI}

Athletes were imaged using a 1.5 Tesla MRI system (GE Healthcare 1.5 T Optima MR360, Chicago, Illinois, USA), and participants lying supine with a surface coil centred at the pubic symphysis and covering the pelvic area. The MRI was performed using routine T1-weighed and T2-weighted and short T1 inversion recovery, that is, STIR sequences (coronal and axial STIR; TR $4000 \mathrm{~ms}$ and TE 42/50 ms;

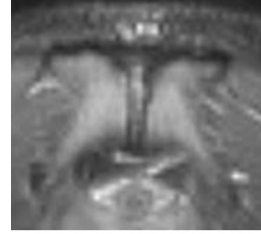

grade I

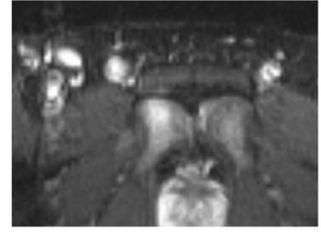

grade II

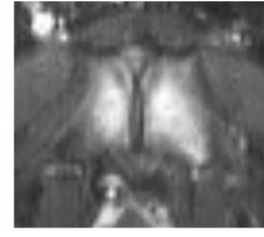

grade III
Figure 2 Pubic bone marrow oedema in MRI was graded as 0 (no oedema), I (mild, $<1 \mathrm{~cm}$ ), II (moderate, $1-2 \mathrm{~cm}$ ) or III (heavy, $>2 \mathrm{~cm}$ ).

slice thickness $5 \mathrm{~mm}$ ). T1-weighted sequence was fast spin echo (FSE) TI 150/170 (coronal T1 FSE and axial T1 FSE; TR $490 \mathrm{~ms}$ and TE minFull; slice thickness $4 \mathrm{~mm} / 5 \mathrm{~mm}$ ). The duration of abdominal MRI was typically $40-50 \mathrm{~min}$. Abnormal findings in the pelvic MRI including BME, an increased signal or tear from the insertion of the adductor, iliopsoas, rectus abdominis or other pelvic muscles were recorded. Pubic BME was graded as 0 (no oedema), I (mild, $<1 \mathrm{~cm}$ ), II (moderate, $1-2 \mathrm{~cm}$ ) or III (heavy, $>2 \mathrm{~cm}$; figure 2). Other recorded pubic MR findings included fatty infiltration around the symphysis, secondary cleft sign and sclerosis/cysts/erosion or disc herniation at the pubic symphysis. ${ }^{15} 18$ Hip pathologies (excessive intra-articular fluid, labrum tears, impingement) were also recorded. All MRI scans were reviewed independently by two experienced radiologists $(\mathrm{SB}$ and $\mathrm{HH})$, blinded to all clinical information on study participants.

\section{Ethics}

Checklist of items of case-control cohort studies (Strengthening the Reporting of Observational Studies in Epidemiology statement; http://www.strobe-statement. org) was followed when reporting the data. All participants provided written informed consent according to the Helsinki Declaration. The project was approved by the local ethical committee on health research and registered into Clinical Trials (ClinicalTrials.gov identifier: NCT02560480).

The statistical analysis was carried out by using SPSS for Windows, V.20.0 (IBM SPSS, Chicago, IL, USA). Statistical evaluation was performed using Fisher's exact test between the groups. The comparison between median VAS scores was analysed using Student's t-test or analysis of variance. A $p$ value of $<0.05$ was regarded as significant for both tests. $95 \%$ CI was reported when evaluating the HAGOS data.

\section{RESULTS}

The prevalence of long-standing groin pain was 15/102 players (annually $7.5 \%$ ). Groin pain was reported in 6,7 and 2 soccer, ice-hockey and bandy players, respectively (table 1$)$. Acute or subacute ( $<30$ days) groin injuries were two times to three times more frequent than longstanding symptoms. Except higher median age of bandy players and higher body mass index in ice-hockey players, duration of groin symptoms, pain scores and training characteristics were rather similar in all teams. The 
Table 1 Characteristics of male athletes with $(n=15)$ or without $(n=43)$ long-standing groin pain

\begin{tabular}{|c|c|c|c|c|c|c|c|}
\hline & Soccer $(n=2$ & & Ice-hockey & $\mathrm{n}=\mathbf{2 3})$ & Bandy $(n=1$ & & \\
\hline & Pain group & Controls & Pain group & Controls & Pain group & Controls & value* \\
\hline Number of athletes & 6 & 16 & 7 & 16 & * & 11 & 0.6011 \\
\hline Age (years, median $\pm S D$ ) & $23 \pm 5.1$ & $22 \pm 2.2$ & $27 \pm 7.4$ & $25 \pm 6.5$ & $31 \pm 0.7$ & $28 \pm 3.2$ & 0.0033 \\
\hline $\mathrm{BMI}\left(\mathrm{kg} / \mathrm{cm}^{2}\right.$, median $\left.\pm \mathrm{SD}\right)$ & $23 \pm 1.1$ & $23 \pm 1.4$ & $26 \pm 1.7$ & $25 \pm 2.0$ & $25 \pm 1.8$ & $23 \pm 0.8$ & 0.0116 \\
\hline Symptoms (months, median \pm SD) & $4.0 \pm 1.4$ & - & $3.1 \pm 1.0$ & - & $3.5 \pm 1.6$ & - & 0.4495 \\
\hline Pain scores $(0-100$, median $\pm S D)$ & $5.3 \pm 2.2$ & $0.8 \pm 0.9$ & $5.7 \pm 0.9$ & $1.1 \pm 0.9$ & $6.5 \pm 0.7$ & $0.1 \pm 0.1$ & 0.6502 \\
\hline Right/left/bilateral pain* & $2 / 4 / 0$ & - & $2 / 4 / 1$ & - & $2 / 0 / 0$ & - & 0.4324 \\
\hline Length of training season (months) & 6 & & 4 & & 4 & & 0.82 \\
\hline Length of competing season (months) & 6 & & 8 & & 8 & & 0.8894 \\
\hline Training per week (hours) & $10-15$ & & $12-15$ & & $8-12$ & & 0.6855 \\
\hline Number of games/year: & 40 & & 70 & & 35 & & 0.0017 \\
\hline
\end{tabular}

${ }^{*} \mathrm{P}$ values are distributed between different sports.

$\mathrm{BMI}$, body mass index; VAS, visual analoque score.

annual number of games was almost double in ice-hockey league compared with other contact sports (table 1).

The prevalence of BME was equal in the symptomatic versus asymptomatic players $(8 / 15 ; 53 \%$ vs $20 / 43 ; 47 \%$, $\mathrm{p}=0.799)$. Table 2 shows the prevalence of BME in absolute numbers in soccer $(5 / 6$ vs $8 / 16)$, ice-hockey $(2 / 7$ vs $9 / 16)$ and in bandy (1/2 vs $3 / 11)$ in the symptomatic versus asymptomatic players, respectively. There were no statistically significant differences in the percentage of BME between symptomatic and asymptomatic players.

Long-standing groin pain was related either to adductors $(n=5)$, inguinal canal $(n=6)$, pubic symphysis $(n=6)$ or hip/ iliopsoas area $(n=3)$. Only one symptomatic soccer player had a normal pelvic MRI. All others had multiple abnormalities on their MRI scans (table 3). Adductor tendinopathy and degenerative changes at the symphysis pubis were almost twice as frequent in symptomatic versus in pain-free

Table 2 BME at the pubic symphysis on MRI scans in male athletes with $(n=15)$ or without $(n=43)$ long-standing groin pain in different sports

\begin{tabular}{|c|c|c|c|c|c|}
\hline \multirow[b]{2}{*}{ Group } & \multirow[b]{2}{*}{ BME (\%) } & \multicolumn{4}{|c|}{ Grade of BME } \\
\hline & & 0 & $\begin{array}{l}\text { I (<1 } \\
\mathrm{cm})\end{array}$ & $\begin{array}{l}\text { II (1-2 } \\
\mathrm{cm})\end{array}$ & $\begin{array}{l}\text { III (>2 } \\
\mathrm{cm})\end{array}$ \\
\hline \multicolumn{6}{|l|}{ Groin pain $(n=15)$ : } \\
\hline Soccer $(n=6)$ & $5 / 6(83)$ & 1 & 1 & $\begin{array}{l}2 \text { (1 } \\
\text { bilateral) }\end{array}$ & $\begin{array}{l}2 \\
\text { bilateral }\end{array}$ \\
\hline Ice-hockey(n=7) & 2/7 (29) & 5 & 0 & $\begin{array}{l}1 \\
\text { bilateral }\end{array}$ & $\begin{array}{l}1 \\
\text { bilateral }\end{array}$ \\
\hline Bandy $(n=2)$ & $1 / 2(50)$ & 1 & 1 & 0 & 0 \\
\hline \multicolumn{6}{|l|}{ Controls ( $n=43)$ : } \\
\hline Soccer $(n=16)$ & $8 / 16(50)$ & 8 & 5 & $\begin{array}{l}3 \text { (1 } \\
\text { bilateral) }\end{array}$ & 0 \\
\hline Ice-hockey(n=16) & 9/16 (56) & 7 & 5 & 3 & 1 \\
\hline Bandy $(n=11)$ & $3 / 11(27)$ & 8 & 3 & 0 & 0 \\
\hline
\end{tabular}

BME, bone marrow oedema. athletes (table 3, figure 3). Rectus or iliopsoas pathologies were rare in both groups (table 3). Pelvic MRI revealed three other types of muscular injuries in the symptomatic group (two proximal hamstring injuries in ice-hockey and bandy player, one sartorius injury in soccer). Two control players from the ice-hockey team had an asymptomatic gluteus medius muscle injury and obturator externus injury at MRI. There were lower HAGOS subscales in ice-hockey and soccer players who experienced hip and/or groin pain than in controls (table 4).

\section{DISCUSSION \\ Main outcome}

Our study indicated that an annual prevalence of groin pain was $7.5 \%$. This is less than reported previously in Denmark from subelite soccer teams. ${ }^{19}$ In that study, 695 soccer players from 40 teams (divisions 1-4), some $49 \%$ ( $95 \%$ CI: $45 \%$ to $52 \%$ ) reported hip or groin pain during the previous season. Of these, $31 \%$ (95\% CI: $26 \%$ to $36 \%$ ) reported pain for $>6$ weeks. In another study, 190 players from nine Dutch professional soccer clubs reported severe groin symptoms in $24 \%$ and within-season incidence of groin injury in $11 \% .^{20}$ One retrospective register study from Finland reported groin injuries in $9.0 \%$ of elite soccer players. ${ }^{6}$ The difference in prevalence rates of groin pain between our study and others may be due to different level of studied athletes (elite vs sub-elite levels). Furthermore, season incidence of pain is always lower than whole year incidence.

The present study extends previous reports that positive MRI findings were common in both symptomatic and asymptomatic athletes in contact sports. Reactive changes (oedema) in the symphysis pubis may be as a consequence of overuse or subclinical contact injury particularly in soccer and ice-hockey and very often represent painless findings. ${ }^{12}{ }^{13}$ In bandy players, the direct pelvic contact occurs more seldom than in ice-hockey and therefore groin injuries may be less frequently observed. 
Table 3 Abnormal MRI findings in male athletes with $(n=15)$ or without $(n=43)$ long-standing groin pain in different sports.

\begin{tabular}{|c|c|c|c|c|c|c|c|}
\hline & \multirow{2}{*}{$\begin{array}{l}\text { Total } \\
\text { Pain/control }\end{array}$} & \multicolumn{2}{|l|}{ Soccer } & \multicolumn{2}{|l|}{ Ice-hockey } & \multicolumn{2}{|l|}{ Bandy } \\
\hline & & Pain group* & Controlst & Pain group* & Controlst & Pain group* & Controlst \\
\hline Adductor tendinopathy & $9 / 4$ & 3 & 2 & 5 & 2 & 1 & 0 \\
\hline Fat infiltration at pubis & $9 / 4$ & 4 & 2 & 4 & 1 & 1 & 1 \\
\hline Secondary cleft sign & $8 / 5$ & 4 & 2 & 3 & 3 & 1 & 0 \\
\hline Sclerosis/cysts/ erosion at the PS & $13 / 9$ & 5 & 4 & 7 & 5 & 1 & 0 \\
\hline Disc herniation & $11 / 16$ & 5 & 2 & 4 & 4 & 2 & 0 \\
\hline Rectus tendinopathy & $1 / 2$ & 0 & 0 & 1 & 2 & 0 & 0 \\
\hline Iliopsoas pathology & $2 / 0$ & 1 & 0 & 1 & 0 & 0 & 0 \\
\hline Other muscle pathology & $3 / 2$ & 1 & 0 & 1 & 2 & 1 & 0 \\
\hline Hip pathology & $2 / 1$ & 1 & 0 & 1 & 1 & 0 & 0 \\
\hline
\end{tabular}

*Pain group means athletes with long-standing groin pain.

†Control group means athletes without long-standing groin pain.

PS, pubic symphysis.

Radiographical findings at the symphysis are often observed as reactive sclerosis at the pubic symphysis. ${ }^{13} 21$ A recent 18-month follow-up trial reported that extensive BME on STIR sequences may predict a poor clinical outcome, that is, the athletes with extensive BME recovered less likely to high-level sports activity. ${ }^{22}$ In contrast to this, we found that BME was as common in symptomatic as in asymptomatic players.

Four clinical entities involved in the long-standing groin pain were recently described as pubic-, adductor-, inguinal canal- (with or without rectus abdominis injuries) and iliopsoas-related groin pain. ${ }^{1516}$ The advantage of pelvic MRI is to detect concurrent injuries, as in our study (proximal hamstring, rectus femoris or gluteal muscle tears). Laxity of the posterior wall of the inguinal canal can be studied clinically using finger/coughing test, by ultrasound or by using

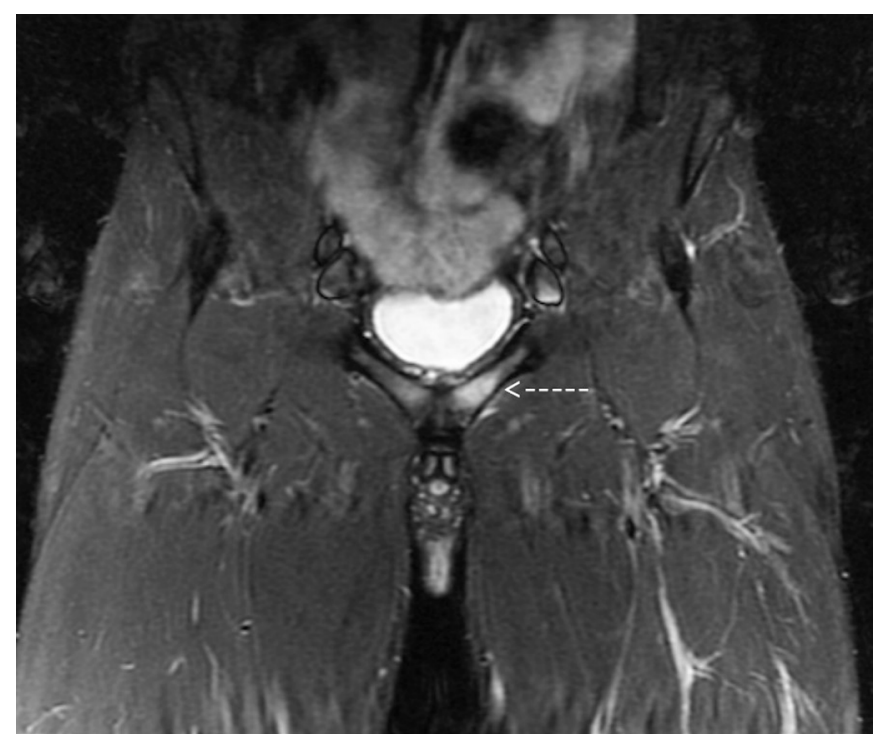

Figure 3 An MRI (STIR sequence) of symptomatic soccer player groin. Grade III bone marrow oedema (arrow) is demonstrated in the left pubic symphysis with an adductor longus injury (bright signal below pubic bone). fast MRI sequence with Valsalva maneuver. ${ }^{23}{ }^{24}$ Asymptomatic MRI findings (tendinosis, calcifications, cortical erosions) are common in athletes and care should be taken when assessing the MRI abnormalities versus groin pain. ${ }^{25}$ We still need well-designed controlled studies to find out how many asymptomatic athletes have adductor-related changes in MRI or weakness of the posterior wall without any relation of groin pain ever. ${ }^{2627}$

Table 4 HAGOS scores in asymptomatic soccer versus ice-hockey players compared with players with longstanding groin/hip pain

\begin{tabular}{|c|c|c|c|}
\hline \multirow[b]{2}{*}{ HAGOS } & \multirow{2}{*}{$\begin{array}{l}\text { Asymptomatic } \\
\text { Median (25th-75th) }\end{array}$} & \multirow{2}{*}{$\begin{array}{l}\text { Groin/hip pain } \\
\text { Median (25th-75th) }\end{array}$} & \multirow{2}{*}{$\begin{array}{l}\text { P } \\
\text { value* }\end{array}$} \\
\hline & & & \\
\hline \multicolumn{4}{|l|}{ Soccer } \\
\hline Pain & $100(100-100)$ & 98 (93-100) & $<0.01$ \\
\hline Symptoms & 89 (77-97) & 78 (76-90) & $<0.01$ \\
\hline ADL & $100(100-100)$ & $98(93-100)$ & $<0.01$ \\
\hline Sport/Rec & $96(92-100)$ & 81 (67-94) & $<0.01$ \\
\hline PA & $100(100-100)$ & $99(94-100)$ & $<0.01$ \\
\hline QL & $98(95-100)$ & 90 (87-98) & $<0.01$ \\
\hline \multicolumn{4}{|l|}{ Ice-hockey } \\
\hline Pain & $100(97-100)$ & 90 (85-95) & $<0.01$ \\
\hline Symptoms & 89 (78-94) & 61 (57-82) & $<0.01$ \\
\hline ADL & $100(97-100)$ & 90 (85-95) & $<0.01$ \\
\hline Sport/Rec & 90 (88-96) & 75 (62-95) & $<0.01$ \\
\hline PA & $98(97-100)$ & 97 (90-98) & $<0.01$ \\
\hline QL & 95 (94-100) & 87 (85-95) & $<0.01$ \\
\hline
\end{tabular}

${ }^{*} \mathrm{P}$ values are distributed between asymptomatic and symptomatic athletes in soccer and ice-hockey.

ADL, activities in daily living; HAGOS, Hip and Groin Outcome Score; PA, participation in physical activity; QL, quality of living; Sport/Rec, sport and recreational activities. 


\section{Limitations}

A limitation of our study was the small number of patients from three different sports. The strengths of this study are the prospective data collection including standardised MRI, as well as a relatively long follow-up period. Furthermore, the MRI scans were blindly reviewed by two experienced musculoskeletal radiologists. Minimum reporting standards on radiology of long-standing groin pain were followed carefully in our study. ${ }^{11} 151827$ The majority of MRI studies have focused on its use for pubic and adductor-related groin pain. ${ }^{1128} 29$ There are very few studies examining the use of MRI in iliopsoas or inguinal canal-related groin pain in athletes. Branci et al developed recently a standardised MRI evaluation protocol (Copenhagen Standardised MRI protocol) for use in athletes with groin pain. ${ }^{1518}$ Ducouret et al utilised axial and coronal T1-weighted, axial-oblique proton density weighted with fat saturation (in symphysis plane), gadolinium contrast enhancement and Valsalva maneuver. ${ }^{24}$ They found that MRI has diagnostic accuracy of $78 \%, 100 \%$ sensitivity, $69 \%$ specificity in evaluating long-standing groin pain when compared with surgical findings. This means that at present, we are not aware $100 \%$ how well pathological MRI correlates to long-standing groin pain and particularly surgical pathologies.

\section{CONCLUSION}

Long-standing groin pain has an annual prevalence of $7.5 \%$. The percentage of BME was $50 \%$ in both symptomatic and asymptomatic players, and it does not correlate well to groin pain.

Acknowledgements We thank the volunteer asymptomatic athletes who participated in the MRI study and our MRI department for high-quality imaging service.

Contributors HP was responsible for the conception and design of the study, recruitment of participants, data collection and drafting. HH and SB contributed to interpretation of the radiological findings and had full access to all data. LR contributed to interpretation of the data and revision of the manuscript. The final manuscript was approved by all authors.

Funding This trial was financially supported by the Competitive State Research Financing of the Expert Responsibility Area of Kuopio University Hospital. Financial support was also received from Orton Orthopaedic Hospital, Helsinki, Finland (the Ministry of Social Affairs and Health grant, 9310/478).

Competing interests None declared.

Patient consent for publication Not required.

Provenance and peer review Not commissioned; internally peer reviewed.

Open access This is an open access article distributed in accordance with the Creative Commons Attribution Non Commercial (CC BY-NC 4.0) license, which permits others to distribute, remix, adapt, build upon this work non-commercially, and license their derivative works on different terms, provided the original work is properly cited, appropriate credit is given, any changes made indicated, and the use is non-commercial. See: http://creativecommons.org/licenses/by-nc/4.0/.

\section{REFERENCES}

1. Litwin DEM, Sneider EB, McEnaney PM, et al. Athletic pubalgia (sports hernia). Clin Sports Med 2011;30:417-34.

2. Nam A, Brody F. Management and therapy for sports hernia. J Am Coll Surg 2008;206:154-64.

3. Gouttebarge V, Veenstra E, Goedegebuure S, et al. Professional football players at risk for non-acute groin injuries during the first half of the season: a prospective cohort study in the Netherlands. J Back Musculoskelet Rehabil 2018;31:15-21.

4. Swan KG, Wolcott M. The athletic hernia: a systematic review. Clin Orthop Relat Res 2007;455:78-87.

5. Rankin AT, Bleakley CM, Cullen M. Hip joint pathology as a leading cause of groin pain in the sporting population: a 6-year review of 894 cases. Am J Sports Med 2015;43:1698-703.

6. Paajanen $\mathrm{H}$, Ristolainen $\mathrm{L}$, Turunen $\mathrm{H}$, et al. Prevalence and etiological factors of sport-related groin injuries in top-level soccer compared to non-contact sports. Arch Orthop Trauma Surg 2011;131:261-6.

7. Smedberg SG, Broome AE, Gullmo A, et al. Herniography in athletes with groin pain. Am J Surg 1985;149:378-82.

8. Minnich JM, Hanks JB, Muschaweck U, et al. Sports hernia: diagnosis and treatment highlighting a minimal repair surgical technique. Am J Sports Med 2011;39:1341-9.

9. Paajanen $\mathrm{H}$, Brinck T, Hermunen $\mathrm{H}$, et al. Laparoscopic surgery for chronic groin pain in athletes is more effective than nonoperative treatment: a randomized clinical trial with magnetic resonance imaging of 60 patients with sportsman's hernia (athletic pubalgia). Surgery 2011;150:99-107.

10. Davies AG, Clarke AW, Gilmore J, et al. Review: imaging of groin pain in the athlete. Skeletal Radiol 2010;39:629-44.

11. Branci S, Thorborg K, Nielsen MB, et al. Radiological findings in symphyseal and adductor-related groin pain in athletes: a critical review of the literature. Br J Sports Med 2013;47:611-9.

12. Daigeler A, Belyaev O, Pennekamp WH, et al. MRI findings do not correlate with outcome in athletes with chronic groin pain. J Sports Science and Med 2007;6:71-6.

13. Lovell G, Galloway H, Hopkins W, et al. Osteitis pubis and assessment of bone marrow edema at the pubic symphysis with MRI in an elite junior male soccer squad. Clin J Sport Med 2006;16:117-22.

14. Paajanen $\mathrm{H}$, Hermunen $\mathrm{H}$, Karonen J. Pubic magnetic resonance imaging findings in surgically and conservatively treated athletes with osteitis pubis compared to asymptomatic athletes during heavy training. Am J Sports Med 2008;36:117-21.

15. Branci S, Thorborg $\mathrm{K}$, Bech $\mathrm{BH}$, Hojlund $\mathrm{BB}$, et al. MRI findings in soccer players with long-standing adductor-related groin pain and asymptomatic controls. Br J Sports Med 2015;49:681-91.

16. Weir A, Brukner P, Delahunt E, et al. Doha agreement meeting on terminology and definitions in groin pain in athletes. $\mathrm{Br} J$ Sports Med 2015;49:768-74.

17. Thorborg K, Branci S, Stensbirk F, et al. Copenhagen hip and groin outcome score (HAGOS) in male soccer: reference values for hip and groin injury-free players. Br J Sports Med 2014;48:557-9.

18. Branci S, Thorborg K, Bech BH, et al. The Copenhagen standardised MRI protocol to assess the pubic symphysis and adductor regions of athletes: outline and intratester and intertester reliability. $\mathrm{Br} J$ Sports Med 2015;49:692-9.

19. Thorborg K, Rathleff MS, Petersen P, et al. Prevalence and severity of hip and groin pain in sub-elite male football: a crosssectional cohort study of 695 players. Scand J Med Sci Sports 2017;27:107-14.

20. Langhout R, Tak I, van Beijsterveldt A-M, et al. Risk factors for groin injury and groin symptoms in Elite-Level soccer players: a cohort study in the Dutch professional Leagues. J Orthop Sports Phys Ther 2018;48:704-12.

21. Lentz SS. Osteitis pubis: a review. Obstet Gynecol Surv 1995;50:310-5.

22. Gaudino F, Spira D, Bangert $Y$, et al. Osteitis pubis in professional football players: MRI findings and correlation with clinical outcome. Eur J Radiol 2017;94:46-52.

23. Vasileff WK, Nekhline M, Kolowich PA, et al. Inguinal hernia in athletes: role of dynamic ultrasound. Sports Health 2017;9:414-21.

24. Ducouret $\mathrm{E}$, Reboul G, Dalmay F, et al. MRI in chronic groin pain: sequence diagnostic reliability compared to systematic surgical assessment. Skeletal Radiol 2018;47:649-60.

25. Pesquer L, Reboul G, Silvestre A, et al. Imaging of adductor-related groin pain. Diagn Interv Imaging 2015;96:861-9.

26. Paajanen H, Montgomery A, Simon T, et al. Systematic review: laparoscopic treatment of long-standing groin pain in athletes. $\mathrm{Br} \mathrm{J}$ Sports Med 2015;49:814-8.

27. Delahunt $\mathrm{E}$, Thorborg $\mathrm{K}$, Khan $\mathrm{KM}$, et al. Minimum reporting standards for clinical research on groin pain in athletes. Br J Sports Med 2015;49:775-81.

28. Mullens FE, Zoga AC, Morrison WB, et al. Review of MRI technique and imaging findings in athletic pubalgia and the "sports hernia". Eur $J$ Radiol 2012;81:3780-92.

29. Bou Antoun M, Reboul G, Ronot M, et al. Imaging of inguinal-related groin pain in athletes. Br J Radiol 2018;91. 